\title{
Configurações
}

Revista de sociologia

19 | 2017

Vária

\section{Ecos de um futuro passado: pós-memórias da nanotecnologia no cinema e videojogos}

Days of Future Past: nanotechnology post-memories in cinema and gaming Échos d'un futur antérieur : post-mémoires de la nanotechnologie au cinéma et aux jeux vidéo

\section{Rui Vieira Cruz}

\section{OpenEdition}

\section{Journals}

Edição electrónica

URL: http://journals.openedition.org/configuracoes/3969

DOI: 10.4000/configuracoes.3969

ISSN: $2182-7419$

\section{Editora}

Centro de Investigação em Ciências Sociais

\section{Edição impressa}

Paginação: 49-71

ISSN: 1646-5075

\section{Refêrencia eletrónica}

Rui Vieira Cruz, «Ecos de um futuro passado: pós-memórias da nanotecnologia no cinema e videojogos », Configurações [Online], 19 | 2017, posto online no dia 30 junho 2017, consultado o 01 maio 2019. URL : http://journals.openedition.org/configuracoes/3969 ; DOI : 10.4000/ configuracoes.3969 
Cruz, Rui Vieira - Ecos de um futuro passado : pós-memórias da nanotecnologia no cinema e videojogos. Configurações, vol. 19, 2017, pp. 49-71

\title{
Ecos de um futuro passado': pós-memórias da nanotecnologia no cinema e videojogos
}

RUI VIEIRA CRUZ*

Universidade do Minho

\begin{abstract}
Resumo
O nosso objetivo consiste em demonstrar que as indústrias culturais, enquanto criadoras de histórias, afirmam-se como autoridades de pós-memória que medeiam, através de artes visuais como o cinema e os videojogos, a construção social dos objetos nanotecnológicos. São diversos os filmes e videojogos representados no futuro em que o narrador/personagem principal apresenta ao espectador/jogador a sua memória traumática de eventos. $\mathrm{Na}$ ótica do espectador/jogador, estes acontecimentos ainda não decorreram, ecoando como um futuro no qual a sequência dos enredos é transmitida como passado. Estudamos as franquias de Terminator e Deus Ex tendo por base metodológica o programa de investigação científico (PIC) lakatosiano, sustentando que as pós-memórias prospetivas são movimentos transgeracionais de construção social em constante diálogo e em constante confronto, nos quais a destruição criativa tecnológica altera/inverte a sua configuração original e apresenta possíveis disrupções narrativas. Concluímos que o papel da nanotecnologia nas indústrias culturais se revela através de ícones e produtos que disciplinam a sua compreensão, refletindo um projeto tecno-político disruptivo, potenciador de uma nova Revolução Industrial.
\end{abstract}

Palavras-chave: nanotecnologia, cinema/videojogos, pós-memórias prospetivas, destruição criativa.

1 Uma ligação ao livro de banda desenhada The Uncanny X-Men (1981) Days of Future Past, traduzido para português (PT-PT, como Dias de um Futuro Passado) em (PT-BR, Dias de um Futuro Esquecido), que aborda elementos de pós-memória.

*Centro Interdisciplinar de Ciências Sociais da Universidade do Minho (CICS.NOVA/CICS-UMinho) e Centro de Estudos em Comunicação e Sociedade da Universidade do Minho (CECS); E-mail: rmvcruz@gmail.com 


\begin{abstract}
Days of Future Past: nanotechnology post-memories in cinema and gaming

Our objective consists in explaining how cultural industries, as storytellers, act as post-memory authorities and mediate, through visual arts such as cinema and video games, on the social construction of nanotechnology objects. Many films and video games take place in a future where the narrator/main character presents the viewer/player to its traumatic memory of the events. In the viewer's/player's perspective, these events have not yet taken place, echoing like a future where the plots' sequence is transmitted as past. We studied the Terminator and Deus Ex franchises according to the Lakatosian scientific research program as a methodological basis. We argue that prospective post-memories are transgenerational movements of social construction, in constant dialogue and confrontation, in which technological creative destruction alters/inverts its original configuration and presents possible narrative disruptions. We conclude that nanotechnology's role in cultural industries reveals itself through icons and products that regulate its understanding, reflecting a disruptive techno-political project, thus enhancing a new Industrial Revolution.
\end{abstract}

Keywords: nanotechnology, cinema/video games, prospective post-memories, creative destruction.

\title{
Résumé
}

Échos d'un futur antérieur : post-mémoires de la nanotechnologie au cinéma et aux jeux vidéo

Notre objectif est de démontrer que les industries culturelles, en tant que créatrices d'histoires, s'affirment comme des autorités de post-mémoire qui sont à la base, à travers d'arts visuels tels que le cinéma et les jeux vidéo, de la construction sociale des objets nanotechnologiques. Plusieurs films et jeux vidéo sont représentés dans un futur dans lequel le narrateur/personnage principal présente sa mémoire traumatique des événements au spectateur/joueur. Dans la perspective du spectateur/joueur, ces événements ne se sont pas encore produits, en faisant écho à un futur dans lequel la séquence des trames est transmise en tant que passé. Nous avons étudié les franchises Terminator et Deus Ex en ayant pour base méthodologique le programme de recherche scientifique lakatosien, soutenant que les post-mémoires prospectives sont des mouvements transgénérationnels de construction sociale en dialogue et confrontation constants, dans lesquels la destruction créatrice technologique modifie/inverse sa configuration originale et présente d'éventuelles perturbations narratives. Nous concluons que le rôle de la nanotechnologie dans les industries culturelles se révèle par des icônes et des produits qui régulent sa compréhension, en reflétant un projet techno-politique disruptif, levier d'une nouvelle révolution industrielle.

Mots-clés: nanotechnologie, cinéma/jeux vidéo, post-mémoires prospectives, destruction créatrice. 
"Premonition... it's just remembering in the wrong direction"

(Doctor Who, The Girl Who Died T9E5)

\section{Introdução}

Tecnologia e progresso têm formado uma aliança concetual e narrativa na qual Estado e indústrias atuam como autoridades de memória no incentivo à produção tecno-industrial. Os eixos da evolução tecnológica tornaram-se objeto de um debate, no qual a definição de nanotecnologia interseta a participação do campo científico, os avanços tecnológicos e os discursos políticos (Jones, $2007)^{2}$. Este ato, que combina o procedimento tecnocientífico e a participação política, criou concetualmente o elemento "nano" como um conjunto de ramos da ciência e tecnologia que trabalham com uma medida entre 1 a 100 nanómetros (nm) (Miller, Serrato, Represas-Cardenas e Kundahl, 2005). Por ser uma nova tecnologia assente numa medida de tamanho, as suas ligações com diversos campos científicos (física, química, engenharias...) e com setores de aplicação (energia, agricultura, robótica, militar) abriu espaço para a imaginação de pós-memórias e para a criação de diferentes cenários futuros.

Assim, visamos demonstrar três argumentos: i) as pós-memórias são construídas e constituem-se como espaços em constante diálogo e confronto; ii) o processo de destruição criativa de implementação nanotecnológica inverte a configuração original das pós-memórias; iii) as indústrias culturais alteram a concetualização tradicional das pós-memórias.

Enquadrados no programa de investigação científico (PIC) lakatosiano (Lakatos, 1967, 1989; Silva, 2009), visamos uma abordagem triangular entre o objeto técnico - nanotecnologia, o conceito operacional -, a pós-memória e os elementos que a manifestam, as indústrias culturais ${ }^{3}$. A partir da moldura de evolução tecnológica neoschumpeteriana ${ }^{4}$ e da concetualização de "destruição criativa", analisamos o modo como a nanotecnologia foi representada e como se compõe um quadro de autoridades de pós-memória em dois produtos das principais indústrias culturais de índole visual (cinema e videojogos): Terminator e Deus Ex. Sustentamos que a pós-memória e os traumas aliados às nanotecnologias suscitam elementos transgeracionais sobre os quais as indústrias culturais constroem identidades e representações da nanotecnolo-

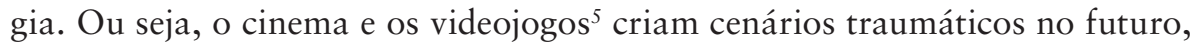

2 Em específico, a partir da sugestão do presidente americano, Bill Clinton, na década de 1990.

3 O programa de investigação científico lakatosiano é composto por núcleo e heurísticas. Se o núcleo permite manter a configuração original de uma teoria, a heurística possibilita a modificação e o cruzamento de teorias sem alterar o núcleo.

4 Schumpeter sistematizou a evolução da tecnologia e da inovação em diferentes ciclos padronizados. 5 Identificamos produtos culturais através da pesquisa da expressão nano (e derivados) em bases de dados comerciais - Amazon - e não-comerciais - IMDb e Metacritic. Dos 29 produtos validados, procurámos um filme e um videojogo que compusessem uma franquia com um mínimo de três entradas 
disciplinando sistemas de crenças, ações e representações no presente. Em cada um dos produtos culturais, procurámos a identificação de variáveis como o tempo cronológico, os eventos decorridos, a forma como a nanotecnologia é destacada na narrativa e o efeito traumático da sua aplicação.

\section{Das pós-memórias retrospetivas às pós-memórias prospetivas (mediadas): diálogos, confrontos e traumas tecnológicos}

Deparamo-nos com uma nova era da memória histórica na qual o passado se funde com o presente (Huyssen, 2003). A narrativa histórica ${ }^{6}$, que teve o "passado" como denominador comum e que outrora serviu para conectar a comunidade e a nação sob a égide de uma herança histórica, apresenta-se fragmentada (Hartog, 2003; Lorenz, 2010). Dessas ruínas, nasce uma história múltipla, regularmente contraditória e fraturada. Autobiografias, diários, testemunhos, documentários, docudrama (Valenzuela e Brandão, 2015), docuficções (Dondero e Bouko, 2015) e redes sociais online (Veltri e Atanasova, 2015) têm posto em causa a "memória de museu" e as suas reificações históricas hegemónicas.

Centrada na diferença geracional, a pós-memória distingue-se da memória

pela distância geracional e da história pela sua profunda conexão pessoal. A pós-memória é uma poderosa e muito particular forma de memória precisamente porque a sua conexão com o seu objeto ou fonte é mediada, não por meio da lembrança, mas através de um investimento imaginativo e criativo... A pós-memória caracteriza a experiência daqueles que cresceram dominados pelas narrativas que precederam o seu nascimento, e cujas próprias histórias são evacuadas/invisibilizadas pelas histórias da geração anterior moldada pelos eventos traumáticos que não podem ser percebidos ou recriados (Hirsch, 1997: 22) ${ }^{7}$.

Na citação de Hirsch (idem, ibidem), denota-se uma distância geracional, uma divisão histórico-temporal entre emissores - possuidores de uma vivência em primeira instância - e recetores de conteúdos, uma geração que não experienciou esses eventos. Estes possuem apenas memórias mediadas, uma forma de conhecimento indireto, pelo que são forçados a recorrer à imaginação para vislumbrar os eventos. Os eventos traumáticos ocupam, contudo, um lugar de destaque no desenvolvimento desta geração, sendo regularmente dominados e

principais, excluindo spin-offs. Procedeu-se à seleção de uma saga cinematográfica e uma de videojogos: Terminator e Deus Ex.

6 Hartog (2003) define o regime de historicidade em dois pilares: em sentido estreito, como uma sociedade trata o seu passado; e em sentido lato, como os regimes de historicidade desenham a modalidade de consciência.

7 Itálicos nossos. 
constrangidos por essas mesmas ocorrências ${ }^{8}$. Argumentamos que a formulação de pós-memória de Hirsch (Hirsch, 2008; Hirsch e Spitzer, 2006, 2009) revela limitações concetuais: a distância intergeracional assenta numa abordagem geracional top-down, do indivíduo mais velho para o mais novo, similar aos storytellers (Gerbner, 2012), e não assume que o inverso é igualmente exequível. Propomos que a pós-memória se constitui como um sistema transgeracional em que é possível às gerações mais novas transmitir a ocorrência de eventos não experienciados pelas gerações mais velhas. Isto coloca os trabalhos tradicionais sobre pós-memórias numa vertente retrospetiva, ou seja, focam-se em eventos passados e re-imaginados pelos indivíduos. Argumentamos que a inovação tecnológica inverte esta tendência, projetando regularmente possibilidades na construção do futuro. Para isso, abordamos conceptualmente a ideia de "pós-memórias prospetivas" (Cohen e Gollwitzer, 2008), ou seja, de eventos que ainda não se realizaram, mas que se inserem na mente dos indivíduos como algo a realizar. A pós-memória prospetiva é o ato de "lembrar de lembrar" (Cohen e Gollwitzer, 2008), um processo cognitivo envolvido na formação e execução de intenções futuras (McFarland e Glisky, 2012). Consiste em projetar a memória (Tenenboim-Weinblatt, 2013) e resulta em ações tão simples como lembrar de fechar a tampa da pasta de dentes, ou mais complexas, como antecipar resultados do conflito entre a Rússia e a Ucrânia aquando a independência da Crimeia em 2014, ou configurar o sucesso em resgatar indivíduos raptados consoante o tempo decorrido. A pós-memória prospetiva assenta em experiências passadas, realizadas por terceiros, e sustenta-se em repetições que moldam o futuro e potenciam algo de inovador num presente imaginado. Este tipo de abordagem de pós-memória, diferenciada da convencional, ocorre já que outras formas de veiculação, assentes nas indústrias culturais e nas tecnologias de informação e comunicação (TIC) (por exemplo, redes sociais online), possuem a capacidade de transmitir conteúdos em registo textual, de imagem e vídeo e fundir diferentes produtores e recetores de conteúdos. Operam como agentes de mediação e propagação para elementos fora da rede (Centola, 2015) nos quais os ecrãs absorvem o choque, filtram e difundem os impactos do trauma (Hirsch, 2008), atuam como um escudo protetor que liga o passado, o presente (e o futuro). Operacionalizámos, por isso, o conceito de "pós-memória prospetiva mediada” (Tenenboim-Weinblatt, 2013), que assenta na experiência direta de eventos não-presenciados e que possibilita a antecipação resultados ou de formas de atuação.

O papel que os mediadores (por exemplo, media, cinema, literatura) desempenham nas construções e nas negociações sociais é o de servir como práticas para contabilizar e marcar o tempo. Contribuem para manter a narrativa e a

$8 \mathrm{O}$ enfoque original localizou-se na forma como as pós-memórias dominavam as vidas dos filhos dos sobreviventes do holocausto (Hirsch, 2008). 
memória sobre ela pela simulação de eventos futuros (Platt, Kamboj, Italiano, Rendell e Curran, 2016), organizar/ritualizar o tempo coletivo e dar pistas perante situações hipotéticas: se acontecer X, podemos atuar de forma Y. Por isso, pós-memórias não são uma posição de identidade. São elementos de poder simbólico e icónico sustentado pelo facto de diferentes gerações possuírem diferentes princípios semióticos. Elementos visuais e textuais ocupam posições diferenciadas na memória e procedem a contributos distintos (McFarland e Glisky, 2012). Por sua vez, as tecnologias e as suas técnicas de previsão incorporam um efeito diferenciador em relação às pós-memórias, visto dependerem destas formas de prospeção. Os ciclos tecnológicos de produção vão tornando, progressivamente, produtos inovadores em produtos obsoletos, em relíquias que ganham o seu espaço em museus ${ }^{9}$. O processador 4004 da Intel, de 1971, embora se constitua como um marco histórico da revolução das TIC, rapidamente $^{10}$ se tornou obsoleto, sendo substituído por modelos de maior capacidade. Esta aceleração tecnológica torna momentos historicamente significantes no desenvolvimento tecnológico em apenas momentos historicamente simbólicos (Martins, 2003). São transformadores, disruptivos, sem, no entanto, representarem um choque cultural imediato. Contudo, a utilização destes de forma regular e sistemática cria um "enxame tecnológico" na produção e no consumo. Esse é o seu contributo para a produção de choques culturais, sendo o mais recente (década de 1970) a revolução das Tecnologias de Informação e Comunicação (Garcia, 2011; Perez, 2007), enquanto a nanotecnologia apresenta potencial para se tornar a próxima revolução tecnológica, substituindo as TIC. Esta (r)evolução tecnológica diferencia-se, assim, de movimentos únicos, como golpes de Estado, revoluções políticas e conflitos bélicos, as tradicionais divisórias nas análises político-históricas que configuram um espaço "antes-evento" e "pós-evento". Argumentamos que o choque cultural da tecnologia se apresenta de uma outra forma: não é focado num único evento transformador, é transitivo.

É a partir desta configuração temporal linear - pré e pós-evento - que surge o interesse das análises sobre pós-memória na temática do trauma, com enfoque regular em transitividades e traumas políticos como, por exemplo, escravatura, holocausto, conflitos militares (Berberich, 2011; Hirsch, 1997; Matsumura, 2008), descartando os traumas tecnológicos. Ocorrências traumáticas nesta esfera são rapidamente interligadas com outros fenómenos de índole sociopolítica, subalternizando a técnica/tecnologia e o seu potencial. O teste aos limites dos sistemas de segurança culminou no desastre nuclear em

9 Museus de tecnologia, museus de videojogos, museus de ciência.

10 A lei de Moore, uma observação feita por Gordon Moore, cofundador da Intel, determina que, a cada 18 meses, é criado um novo processador com o dobro da capacidade e metade do tamanho. Após a criação do Intel 4004, em 1971, sucedeu-se o 8008, em 1972, e a consequente evolução para o 4040 e o 8080 , em 1974. 
Chernobyl (Ucrânia), em 1986, e, mais recentemente, os problemas na central nuclear em Fukushima (Japão), em 2011, afetaram a aceitação popular sobre energia nuclear. As experiências com energia nuclear afetaram também as indústrias culturais: o filme Stalker (Tarkovsky, 1979) atuou como reação ao desastre de Chelyabinsk, em 1957, e como premonitório em relação a Chernobyl, de $1986^{11}$.

Portanto, conhecimento situado gera memórias situadas. E as pós-memórias ressentem-se disso. Nos desastres nucleares, temática relacionada com a discussão política e descaracterizada da sua componente técnica, as pós-memórias coletivas de europeus e japoneses na sua compreensão são distintas das de países não afetados, da mesma forma que judeus e nazis não possuem as mesmas pós-memórias coletivas referentes à $2^{\text {a }}$ Guerra Mundial. Outros exemplos de traumas tecnológicos foram as falhas técnicas que provocaram as fissuras que levaram ao acidente na barragem de Bangqiao (China), em 1975, conduzindo à morte de mais de 150 mil pessoas e a mais de 10 milhões desalojados. O acidente químico de Bhopal (Índia), em 1984, gerado pelo desgaste das condutas de gás, comportou a morte de entre 3 a 16 mil indivíduos, afetando mais de 500 mil. Os apagões em Northeast (EUA), em 1965 (falha no sistema de distribuição da rede) e em 2003 (bug no software), deixaram mais de 30 e 55 milhões de norte-americanos sem energia elétrica, respetivamente, levando a motins e assaltos ${ }^{12}$.

Esta criptomnésia ${ }^{13}$ social, ou melhor criptomnésia sociotécnica, é regularmente fundamentada pelos estudos centrados nos impactos da tecnologia (Ball, 2010, Gordijn, 2005, Grinbaum, 2010, Laan, 2010). A tecnologia afeta comportamentos e dinâmicas sociais, mas a sua construção é amplamente configurada como um deus ex machina - como algo que, simplesmente, aparece na vida social. Esta limitação ignora, por exemplo, que a transição do sistema industrial fordista-keynesiano para as TIC fez emergir novas figuras, como os hackers. Imaginados como "piratas", foram-lhes atribuídas capacidades de alterar o posicionamento de mísseis ou invadir estabelecimentos bancários a partir de casa. Antagonicamente, tidos como libertadores, os hackers revelam novas formas de lutar contra um sistema opressivo e compõem figuras como as de cyberhackers e biohackers. A aceitação ou rejeição tecnológica (e das suas figuras) potencia um imaginário social e culmina na demonstração de que não

11 Personagens como Godzilla, lançado originalmente no Japão, em 1954, e zombies, como os recriados no filme The Night of the Living Dead, de 1968, compõem algumas das figuras nascidas dos riscos associados ao desenvolvimento tecnológico e aos perigos de contaminação nuclear resultante da execução de testes militares com energia atómica.

12 A energia elétrica, tida como garantida desde as "Guerras das Correntes", no final do século XIX, quando Nikola Tesla criou o sistema de distribuição de corrente alternada, alertou um país para os esforços diários necessários para a garantir.

$13 \mathrm{O}$ ato de experienciar uma memória como algo novo. 
há relações de poder e de domínio sem resistências (Foucault, 2001; Krips, 2010).

No entanto, a (pós)memória é falível. Os testemunhos exigem uma contínua desconstrução dos elementos criados pelos participantes e dos recetores externos. Exige também um trabalho de memória (Ricoeur, 1984, 1986) para ser regularmente lembrada. O léxico do imaginado entrou na realidade discursiva através destes trabalhos de memória, triangulando tecnologias, pós-memórias e indústrias culturais. Este sistema "torna o estranho familiar" (Gil, 2002). Referências culturais como o Big Brother (do livro 1984, de George Orwell) servem de exposição a modelos de vigilância, sistemas de autoridade e programas televisivos. Na nanotecnologia, a imagem da miniaturização destaca-se pela presença de minissubmarinos que atacam infeções e combatem vírus. Portanto, as pós-memórias são espaços construídos em constante diálogo e confronto. São recursos escassos, mas plurais, que competem umas com as outras. Simultaneamente multidirecionais (Rothberg, 2009) e competitivas, as pós-memórias não são somente história narrada ou oficial: são poesia, literatura, fotografia, cinema ou videojogos. As memórias coletivas estruturam-se, portanto, como "pós-memórias hibridas”. Existem perigos quando as pós-memórias são reificadas em formatos como as histórias de vida, os flashbacks, as narrativas mitificadas ou um cérebro central (Martins, 2006): os perigos da histórica única (Adichie, 2007) do ponto de vista do domínio hegemónico ou exclusivista. Ao selecionar apenas pedaços da memória (Orlando, 2016), corre-se o risco de tornar estática uma prática dinâmica. Desta ação disciplinadora, surgem pós-memórias coletivas que desempenham um papel fulcral no plano da política e sociedade:

As imagens da Guerra do Vietname limitam o apoio às atividades militares americanas; memórias do período nazi restringem a política externa e doméstica alemã; lembranças do período ditatorial moldam as atividades dos regimes de transição e de pós-transição, desde a Europa de Leste à América Latina; e Watergate tornou-se o ponto de referência perene para todos os subsequentes escândalos em Washington (Olick, 1999: 333).

\section{Evolução cíclica: pós-memórias e destruição criativa}

Schumpeter (2003: 81), um dos principais estudiosos no estabelecimento de ciclos económicos, desenvolveu o conceito de destruição criativa e definiu-o como uma mutação industrial que "incessantemente revoluciona a estrutura económica a partir de dentro, incessantemente destruindo o antigo, incessantemente criando um novo". Deste conflito entre o "novo" e o "velho" resulta 
uma vantagem competitiva que cria uma vaga de imitadores ${ }^{14}$ e somente aí, pelo resultado da competição, é gerada uma corrida de inovação que irá destruir as empresas, sistemas de produção ou tecnologias que não são capazes de a acompanhar: "este processo de destruição criativa gera a entrada de novos inovadores e a saída dos velhos inovadores" (Aghion e Howitt, 2006: 275). Esta força de destruição criativa torna o outrora novo em obsoleto, dando origem a novos processos, novas tecnologias, novas realidades e novas mundividências (Schumpeter, 1947; Shionoya, 2007).

Na teoria cíclica, o processo de evolução é padronizado, a sua direção não o é (Balabkins, 2003). É através desta metamorfose que a destruição criativa se alia às pós-memórias prospetivas. A destruição criativa pode "destruir" velhas tecnologias, mas não destrói as memórias que os indivíduos têm delas e os traumas que possam ter gerado. As gerações anteriores dominavam tecnologias que, entretanto, se tornaram obsoletas, enquanto as novas gerações, desligadas deste processo, incorporaram as novas tecnologias sem a assimilação das anteriores. Este trabalho de memória e de partilha suscita um diálogo transgeracional assente no trauma, mas numa dupla aceção do "risco": aventurar, de forma a suplantar o trauma; ou recear, rejeitando o "novo". Por isso, uma nova tecnologia pioneira, por exemplo, nanotecnologia, não se limita à criação de novas indústrias produtivas, mas também de sistemas políticos e culturais, económicos e sociais ${ }^{15}$. O sucesso num setor tecnológico molda a sua integração em outras áreas como as indústrias culturais e atrai a atenção para a consideração do que é uma inovação desejável:

O sucesso em qualquer sector inicialmente afeta a organização social ao elevar a posição do 'líder' bem-sucedido e por lhe conferir...uma base de poder social. Sucesso em qualquer sector influencia os valores sociais em geral, aquilo que chama atenção, o que está a ser considerado como bom ou desejável. E em todos estes sucessos em qualquer sector de atividade social eventualmente terá um efeito formativo ao longo de todos os sectores da vida social. (...) Desconectamos isto de um conjunto rígido de cadeias causais e devolvemos-lhe à vida, novamente. E nesta compreensão holística do desenvolvimento cultural, a economia também tem o seu espaço próprio (Schumpeter, 2002: 431) ${ }^{16}$.

14 A distinção schumpeteriana entre i) invenção (o ato de criação de um novo produto), ii) inovação (o ato de comercializar o novo produto ou forma de produção) e iii) imitação (o ato de espalhar o produto ou forma de produção através diversos sectores) (Ruttan, 2010; Schumpeter, 2003; Shionoya, 2004; Swedberg, 2002).

15 Isto não significa que a tecnologia seja o prime-mover social (a infraestrutura no quadro da terminologia marxista). Demonstra que a tecnologia é afetada pelos sistemas sociais, políticos e económicos, mas que afeta tais sistemas. Alguns exemplos: o automóvel alterou o sistema de transportes; ii) a eletricidade mudou o sistema social; iii) a internet modificou a produção cultural; e estas invenções moldaram o sistema económico, social, político e cultural.

16 Itálico nosso. 
Tecnologias e pós-memórias necessitam de uma base social comum, em que a inovação resulta num processo que se estabelece entre diversos agentes: “A competição da (...) nova tecnologia (...) age, não apenas quando já existe, mas também quando é meramente uma ameaça sempre presente. Disciplina antes de atacar" (Schumpeter, 2003: 84-85)17. Este disciplinamento ocorre, sobretudo, de duas formas: canibalização (Kauffman e Techatassanasoontorn, 2012; Osarenkhoe, 2010) e Brand Value over Time (BVoT) (Wasserman, 2015).

O efeito de canibalização é uma estratégia assente no processo de destruição criativa (Herrmann, Sprott e Schlager, 2015; Peng, Agarwal, Hosanagar e Iyengar, 2014), quase sempre presente ${ }^{18}$ pela substituição regular de hardware e software (Marchand e Hennig-Thurau, 2013). Por sua vez, o BVoT sustenta uma ligação consistente entre criadores, produtos e consumidores (Holt, 2002; Wasserman, 2015). Os produtos tecnológicos substituem-se, mas mantêm uma coerência previsível (Motohashi, Lee, Sawng e Kim, 2012; Nagar, 2015). Esta capacidade de reprodução ${ }^{19}$ constante, na qual a escala temporal transforma o "novo" em "velho", configura o estado-da-arte tecnocientífico (Schmidt, 2008). Visto que as tecnologias e as (pós)memórias não existem num vácuo, imaginar a nanotecnologia a partir das pós-memórias mediadas cria um conjunto de pistas para a real aplicabilidade. É aqui que as indústrias culturais disciplinam e imaginam a aplicação das tecnologias e fomentam novas pós-memórias.

\section{Objeto artístico nas indústrias culturais: representações, unidade e efeito Diderot}

As representações sobre a arte não são estáticas e sofreram alterações, inicialmente delimitando espaços de fronteira entre alta arte, elitista, e baixa arte, popular (Bauman, 2007, 2010). Essas fronteiras apresentam-se atualmente mais esbatidas e integradas (Bauman, 2007). A fluidez da produção cultural promove o acesso a bens culturais através de diversos formatos (por exemplo, Internet, redes sociais online), a partilha e a promoção informações similares por diferentes indivíduos (Bauman, 2009, 2010). Porém, a forma como a sociedade industrial organizou a sua base tecnológica tende para um totalitarismo (Marcuse, 1977, 2002) não em termos políticos, mas na forma como os produtos fazem parte integrante da identidade dos indivíduos e dos grupos sociais: “as pessoas reconhecem-se nos seus produtos" (Marcuse, 2002: 11). As indústrias culturais que operam através da imagem (por exemplo, cinema, videojogos) promovem uma relação social entre indivíduos mediada pelas imagens, atuando como uma forma de fetichismo da mercadoria (Debord, 1995).

17 Itálico nosso.

18 A título de exemplo, os cartuchos usados no sistema da NES (1985) não são compatíveis com os da tecnologia seguinte, a SNES (1990). Os cartuchos do modelo seguinte, N64 (1996), também não eram compatíveis com os anteriores.

19 A lei de Moore atua também como um princípio demonstrável da destruição criativa e das formas de canibalização comercial. 
É possível perceber as categorias de assimilação e as formas ideológicas de promoção de conteúdos. As indústrias culturais possuem a função de uniformização, de diluição dos fenómenos, um controlo fantasmagórico do desejo (Cruz, 2002; Miranda, 2002), mas também uma função educativa e ilustrativa, servindo de mínimo cultural comum, que transmite uma formação ilustrativa de unidade, uma representação de uma realidade. O cinema assenta numa visualização passiva em que o indivíduo assiste ao filme (Zimmer, 2011), mas atua como ilusão, integrando-o nele, fazendo-o crer que faz parte dele (Allen, 1993). Por sua vez, os videojogos permitem interatividade com a narrativa e a utilização diferenciada de materiais nanotecnológicos por parte dos jogadores (Cruz, 2013). Esta ideia de unidade, o efeito Diderot ${ }^{20}$, molda o comportamento das indústrias culturais e assenta em duas premissas: i) os produtos consumidos têm em vista a identidade do consumidor; ii) e a introdução de um novo, mas atípico produto catalisa um processo de consumo em espiral: "Eu era o mestre absoluto das minhas velhas vestes. Eu tornei-me escravo das minhas novas" (Diderot, 2005: 2). Se, na primeira parte desta frase, está implícita a habituação numa forma quase alienante e reificante, na segunda, o objeto toma o controlo e fomenta uma transformação comportamental. A ação social passa a ser mediada pelos produtos (fetichismo da mercadoria), fundindo a pós-memória (enraizamento de uma narrativa) e a destruição criativa na incorporação nanotecnológica. O "novo" reflete o desconhecido, mas é através do fetichismo da mercadoria que "o novo" é transformado em algo reificável, expectável, conhecido e agregador, mediado pelas indústrias culturais. Não transforma somente o "novo" em "velho". Transforma a sua base: o processo.

As indústrias culturais disciplinam a inovação resultante da destruição criativa, em que novos meios aumentam o ritmo de adesão e os seus impactos (Martins, 2003). Veículos como a rádio, a banda desenhada, o cinema e a Internet demonstraram, junto dos seus públicos ${ }^{21}$, um conjunto de possibilidades imaginadas do potencial da tecnologia, contribuindo para a sua internalização/domesticação (Pereira, 2002) e para fomentar uma aceleração temporal (Hassan, 2010; Rosa, 2003; Violante, 2002) que resulta numa fusão entre passado-presente-futuro (Dawesar, 2013) e entre o físico e o virtual ${ }^{22}$. Na banda desenhada, as interpretações das relações entre judeus e nazis retratadas em

20 Quando Diderot procedeu à aquisição de um novo manto, notou que nenhum dos antigos produtos que detinha estava em consonância com o seu novo produto. Como tal, procedeu à substituição das suas antigas posses, levando a um consumo em espiral de novos produtos que estivessem em sintonia com o seu novo manto.

21 A título de exemplo, a leitura radiofónica de War of the Worlds gerou uma onda de pânico nos Estados Unidos, em 1938; o combate público do Superman contra o Klu Klux Klan, numa série de 16 episódios intitulados "Clan of the Fiery Cross", transmitidos na rádio e na banda desenhada durante a década de 1940, gerou uma onda de consciencialização de crianças e adolescentes contra o KKK.

22 Um vídeo deixado numa plataforma de distribuição online (por exemplo, o Youtube) pode ser consultado anos após a sua produção (futuro), transmitindo uma ideia do seu criador no tempo presente, que, entretanto, se tornou passado. 
MAUS, de Art Spieglman, apresentam uma analogia animal entre gatos e ratos e vislumbram a forma como um filho imaginou a vida dos pais num campo de concentração nazi (Hirsch, 1993). A Guerra Fria e os impactos da energia nuclear foram alvo de estudo da formação identitária da editora de banda desenhada Marvel Comics e do modo como esta editora promoveu estas temáticas junto de crianças e adolescentes (Genter, 2007). Também o cinema tem contribuído para a reflexão das pós-memórias traumáticas, incidindo em tópicos tão diversificados como as reclusas marroquinas (Orlando, 2016), os períodos de fome na Ucrânia na década de 1930 (Gurga, 2012, 2014), os contributos da União Soviética para a memória do Terceiro Reich (Berghahn, 2006), a reflexão intergeracional sobre familiares aliados ao regime nazi (Berberich, 2011), a sinofonia após a revolução cultural (Chen, 2015), os laços sociais na zona desmilitarizada coreana (Choe, 2013) e os impactos e reflexões sobre a bomba atómica no Japão (Matsumura, 2008).

Aliada ao trauma, está a figura do fantasma quase omnipresente que paira constantemente sobre a realidade. Um evento torna-se, assim, numa figura social que desempenha um papel numa tomada de decisão. Uma demonstração do peso fantasmagórico da pós-memória histórica ocorre na representação jurídica de Holodomor, na Ucrânia - uma tentativa de extermínio pela fome durante 1932-33 -, quando, em 2006, o governo local emitiu uma lei que estabeleceu que esquecer ou negar o que considerou um genocídio contra o povo ucraniano era passível de ação jurídica. Este genocídio foi regularmente lembrado pelo cinema ucraniano que desempenhou um "trabalho de memória" para não esquecer o evento/trauma (Gurga, 2014). As obras Prey (Crichton, 2006) e The Diamond Age (Stephenson, 1995) expandiram este efeito fantasmagórico da pós-memória para a esfera da nanotecnologia. Crichton (2006) aliou o fantasma ao invisível e secundarizou a definição científica de nanotecnologia para incidir sobre as suas aplicações, traumas e riscos. A temática centrada na ameaça à saúde e nas representações em torno da concetualização de Drexler (1990) de um cenário de gosma cinza no qual os nanorobots entrariam nas corrente sanguíneas e começariam um ciclo interminável de replicação constante serviu para estes autores abrirem espaço à fundamentação das suas narrativas. Na banda desenhada, Livewires (Warren e Mays, 2005) e The Authority (Ellis e Hitch, 1999) apresentam ao leitor um mundo repleto de possibilidades técnicas, mas também de traumas e conflitos ${ }^{23}$. De forma distinta, o filme e os estudos sobre El Laberinto del Fauno (Del Toro, 2006) relembraram o peso da herança histórica deixada pelo franquismo em Espanha,

23 As ciências sociais ancoraram a nanotecnologia a uma série de narrativas históricas e mitos, também alguns deles extraídos das indústrias culturais. De futuros utópicos (Roco, 2007; Smalley, 2007) a futuros distópicos (Gordijn, 2005), do mito do Golem (Grinbaum, 2010) às frankenfoods (Ball, 2010; Laan, 2010), da caixa de Pandora (Dupuy, 2010) ao socialismo científico marxista (Toumey, 2011), as narrativas assentes em (pós)memórias têm contribuído para as representações da produção nanotecnológica. 
mas centrados na sua conclusão: a importância de esquecer e matar o passado (Miles, 2011). Esta destruição criativa de pós-memória - o esquecimento ativo do período ditatorial - não se configurou enquanto ato de alienação, mas em trabalhos de memória de ativamente apagar uma ideologia, em não permitir que aquele passado traumático influencie o futuro (Gómez-Castellano, 2013). Trata-se de projetar um futuro pela construção do presente, sem a intervenção de um passado específico. Os discursos do que era - o passado - fundem-se com a execução real do presente e com as potencialidades possíveis no futuro.

Os espaços virtuais/imaginados comprimiram a narrativa na História. Deixamos de perceber a narrativa numa evolução linear de passado-presente-futuro para passarmos a ter indivíduos presentes em diversos espaços em diversos tempos - digital now (Dawesar, 2013). A ficção na literatura, cinema, séries televisivas e videojogos tem consistentemente avançado com cenários (oscilando entre possível, plausível e irrealista) que moldam e acostumam os seus recetores a novas vagas tecnológicas. A pós-memória não é somente o passado tornado presente. As memórias não são blocos de legos que se vão encaixando e acumulando em que o peso do passado determina o presente. Por isso, a pós-memória prospetiva (Kliegel e Martin, 2003) evita um efeito "ovo de colombo": perceber a solução após nos ser revelada. Ao invés, reforça a criação e reflexão de novos elementos narrativos, em diferentes formatos, apresentando diversos choques e incongruências.

\section{Nanotecnologia e traumas tecnológicos: dos diálogos e conflitos das pós-memórias, à destruição criativa e efeito Diderot em Terminator e Deus Ex}

Terminator e Deus Ex são duas franquias ${ }^{24}$ que demonstram como as indústrias culturais criam "pós-memórias prospetivas mediadas” da nanotecnologia. Ao centrar as suas narrativas no futuro, criam representações de eventos traumáticos - guerras, genocídios e conflitos sociais globais - que ainda não aconteceram. Este futuro imaginado apresenta-nos uma geração mais nova a demonstrar aos espectadores, a geração mais velha, a sua visão do trauma como se fosse o presente. A representação do conflito e do trauma assenta na nanotecnologia e ambos os produtos apresentam uma dicotomização acentuada: Terminator foca o binómio entre humanos e máquinas e Deus Ex entre bio-humanos e trans-humanos. No primeiro, a nanotecnologia é apresentada do lado das máquinas como uma característica que as torna superiores e como obstáculo que os humanos têm de superar. No segundo, a nanotecnologia é

24 Terminator é composto por cinco filmes: i) The Terminator (1984); ii) Judgment Day (1991); iii) Rise of the Machines (2003); iv) Salvation (2009); v) Genisys (2015). Deus Ex é composto por quatro videojogos: i) Deus Ex (2000); ii) Deus Ex: Invisible Wars; iii) Deus Ex: Human Revolution; iv) Deus Ex: Mankind Divided. 
incorporada nos humanos e transmite uma ideia de evolução: separa o humano biologicamente determinado, o bio-humano, do trans-humano, alguém que superou a sua condição biológica, mas é heteronomamente controlado pela tecnologia (Garcia, 2003).

Terminator é narrado do futuro para o passado/presente e apresenta três períodos temporais: i) 2018, a era em que as máquinas/inteligências artificiais entram em guerra declarada com os humanos; ii) 2029, em que as máquinas disputam as batalhas/eventos principais com os humanos; e iii) os períodos temporais em que cada filme decorre. Ancorado narrativamente no ano de 2029, os humanos lançam uma ofensiva global contra a inteligência artificial (IA) Skynet, resultando na vitória dos humanos no conflito contra as máquinas que se desenrolava desde 2018, o ano em que as máquinas lançam uma ação global de genocídio contra os humanos. São regularmente focados dois destinos de evolução tecnológica. No primeiro caso, a proximidade tecnológica do modelo T-800 com os humanos torna-o num objeto sapiente e senciente. O seu sacrifício pessoal serve como metanarrativa da aproximação da tecnologia com o que ainda é humano (Kline, 2009; Muri, 2003) e representa um ponto no qual a tecnologia foi criada por humanos com o objetivo de os servir. No segundo, os modelos seguintes, por exemplo, T-1000, T-X e T-3000, demonstram a perda da sua humanidade, uma transformação numa tecnologia que deixa de ser produzida por humanos e de os servir. Nesta nova direção de evolução, os humanos tornam-se desnecessários e as máquinas são capazes de processar os meios mais eficientes para cumprir os seus objetivos.

A imagem do humano obsoleto e objeto de extermínio presente na saga Terminator está fortemente imbricada na metáfora do holocausto: “a decisão de exterminar, não apenas um grupo enquanto tal, mas também todos os seres humanos classificados como pertencentes a esse grupo, até ao último" (Ribeiro, 2010: 10). O processo de reconfiguração substituiu os nazis pelas máquinas e fundiu os judeus na conceção de humanos. Contudo, há reformulações narrativas: os elementos obsoletos derrotam os inovadores, ou seja, os humanos derrotam as máquinas e um velho modelo T-800 contribui derrotar as tecnologias estado-da-arte, os modelos T-1000, Terminatrix e T-3000. O conflito militar e a política de extermínio geraram um efeito traumático fantasmagórico sempre presente, no qual os filmes configuram as ações do passado e do presente como causadoras do conflito de 2029 e as ações de pós-memória transmitidas para os públicos a partir da narração dos humanos ${ }^{25}$. Neste desenrolar, o testemunho e a memória são subordinados à reconstrução histórica e ao apuramento dos factos (Ribeiro, 2010). A narrativa segue um fluxo próprio no qual a ação de memória dos protagonistas serve apenas como enredo explicativo dos eventos maiores. A oscilação entre passado-presente-futuro representa a hibridez dos 
trabalhos de memória e apresenta, numa mesma narrativa, diversas versões das mesmas personagens.

Por sua vez, Deus Ex apresenta ao jogador um futuro distópico no ano de 2052, colocando-o no controlo de um agente nano-aumentado com capacidade super-humanas, no qual os elementos de poder económico estão diretamente relacionados com as atribuições físicas. A humanidade foi atingida por um vírus cuja vacina não consegue ser produzida em quantidade suficiente para todos e é apenas distribuída pelos indivíduos vitais para o funcionamento societal. O nível de desigualdade social manifestado conduziu a motins à escala global entre dois grandes grupos: bio-humanos e nano-melhorados. Deus Ex Invisible Wars ocorre em 2072, 20 anos após Deus Ex, e apresenta ao jogador um mundo em conflito no qual quatro fações entraram em conflito: i) isolacionistas, que fortificaram as suas cidades e limitaram o acesso de refugiados; ii) fações religiosas, que lutam pelo fim da nanobiomodificação; iii) fações que se pretendem expandir globalmente e gerar uma raça somente de trans-humanos; e iv) uma fação que pretende um patamar de igualdade global e a criação de um mínimo de biomodificações comum a todos os (trans)humanos. O último dos três títulos, Deus Ex: Human Revolution, ocorre em 2027, e atua como prequela. O foco da narrativa nas vantagens/desvantagens do trans-humanismo, na dimensão bélica do conflito e nas desigualdades sociais da engenharia genética revela o nascimento dos acontecimentos traumáticos dos outros dois jogos. Entre personagens que pretendem ultrapassar a condição bio-humana através da nano(bio)tecnologia e aqueles que pretendem a evolução natural da espécie, o foco nos nanomelhoramentos como estado-da-arte, apenas acessível a um nicho minoritário em locais similares a supermercados genéticos (Garcia, 2006), despoletou um nível de desigualdade social difícil de controlar. A introdução da nanotecnologia neste leque serviu para criar um terceiro grupo (nano-melhorados) e veio demonstrar que os quadros de evolução económica, social e cultural não foram capazes de acompanhar a evolução tecnológica.

Uma crítica manifesta-se de forma latente em Terminator. A IA Skynet, resultado da ligação da tecnologia aos conflitos militares humanos, passa a encarar os humanos como um vírus que tentam erradicá-la. Nesta ótica, as máquinas lutam pela autopreservação e revelam-se como seres sapientes que, progressivamente, perdem a capacidade senciente de compactuar com os humanos, escalando o nível de violência, normalizando-a, numa ação similar à banalização do mal (Arendt, 2006). Dependem unicamente da lógica, operam a partir de um cérebro central (Hayles, 2006) e visam eliminar o problema que compete com o seu sentido evolutivo como sendo apenas mais uma tarefa a cumprir. Também em Deus Ex todas as fações lutam pela autopreservação das suas formas de vida, da sua evolução e das suas culturas dentro de um grande binómio: abraçar ou rejeitar a revolução nanotecnológica. 
Em Terminator, a nanotecnologia é incorporada na robótica, e as máquinas, tecnologicamente mais fortes, competem ativamente com os obsoletos humanos. A missão das máquinas passa por se camuflarem como humanos e passarem despercebidas no meio destes: conferir uma ideia de unidade na sua diversidade. Em Deus Ex, a nanotecnologia incorpora-se nos humanos e fomenta o trans-humanismo, alterando a função social da nanotecnologia. Esta dicotomia entre bio-humano e trans-humano constata-se através da indumentária. Os inovadores trans-humanos (representantes da revolução tecnológica) foram artisticamente incutidos de um espírito Renascentista encaixado no quadro estilizado cyberpunk (imagem 1)enquanto os bio-humanos são representados com jeans e $t$-shirt (imagem 2) algo mais similar aos códigos de vestuário atuais, reproduzindo uma visão estática da evolução e não uma revolução. O processo de destruição criativa e efeito Diderot adensa-se: no momento em que a humanidade subiu um degrau na evolução, a obrigação de ser constituída apenas por bio-humanos torna-se desnecessária. No momento em que os trans-humanos abarcaram algo novo demonstraram a necessidade de alterar as suas vivências e incorporar novos bens (o vestuário e estilos de vida) que conferissem uma unidade à sua decisão. O objetivo da indumentária, nos trans-humanos, consiste em distinguir-se dos humanos, o que se diferencia de Terminator. Neste franchise cinematográfico, a necessidade da as máquinas de se assemelharem aos humanos para otimizar a infiltração militar conecta-se com a imagem do cyborg (Haraway, 1985; Shields, 2006) e a forma como aquelas se constituem como agentes híbridos (imagem 3$)^{26}$.

Imagem 1. Trans-humanos (indumentária)

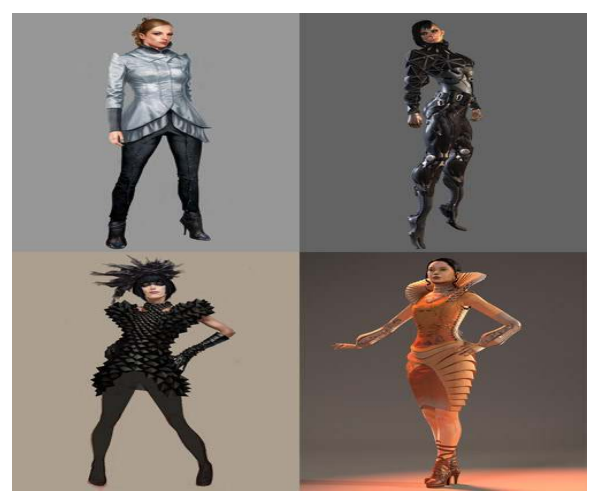

Fonte: Square Enix.
Imagem 2. Bio-humanos (indumentária)

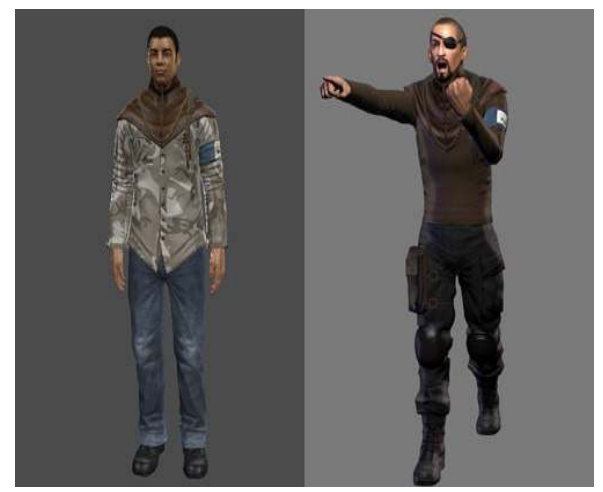

Fonte: Square Enix.

26 No entanto, não podemos deixar de destacar que a cada Terminator foi atribuído um elemento de género, centrado no binómio masculino-feminino. 
Imagem 3

Diferentes Terminator e a sua semelhança Humano-Máquina

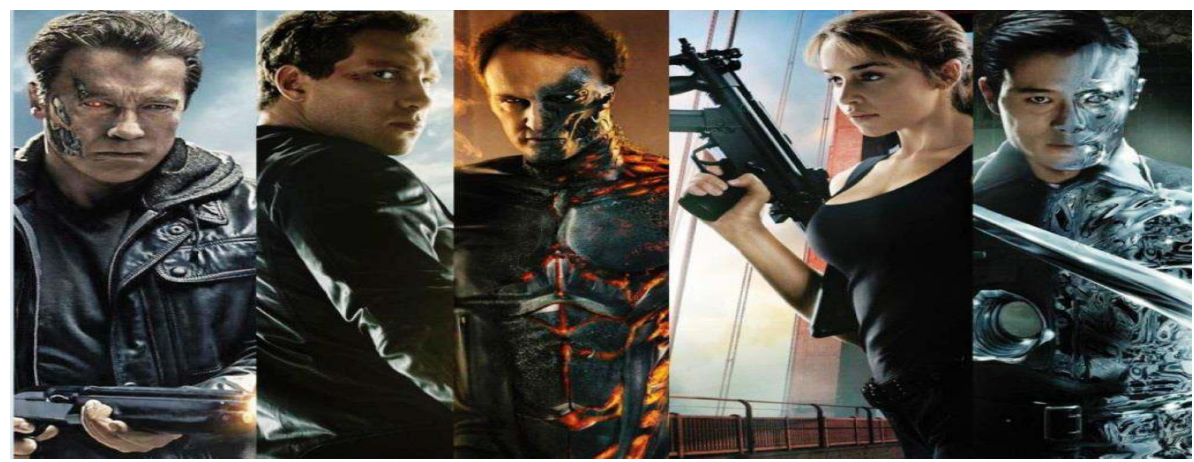

Fonte: Poster promocional da Paramount Pictures.

O objetivo das máquinas consiste em erradicar os humanos, mas o seu efeito de unidade distingue-se de Deus Ex, uma vez que a ideia de unidade reside na forma como as máquinas comunicam entre elas e não na forma como elas se assemelham aos humanos. Deus Ex e Terminator apresentam, por isso, duas formas distintas do processo de destruição criativa e do efeito Diderot, sem no entanto, alterarem as bases fundamentais dos conceitos.

Um elemento diferenciador reside na distinção entre os humanos e as máquinas e na suscitação do binómio "Nós" e "Eles", identidade e alteridade. Em Terminator, os humanos, por força das circunstâncias, unem-se para combater as máquinas, também elas unidas em torno da IA Skynet. A viagem no tempo foi um resultado do trauma do conflito bélico travado entre as duas fações. Em Deus Ex, esta conceção de viagem de tempo foi substituída pela ação do jogador, visto que ele pode escolher com que fação quer cooperar e que mundo quer vislumbrar no futuro. O conflito está sempre presente, não havendo uma dicotomia entre "bons" e "maus", apenas entre bio-humanos e trans-humanos. A humanidade apresenta-se, por isso, dividida.

\section{Notas finais}

As pós-memórias são narrativas tendencialmente mitificadas que ora discutem, ora compactuam com a história oficial. Ao centrarmos a análise nas pósmemórias prospetivas mediadas no cinema e nos videojogos, constatamos que ambas apresentam diversos trajetos evolutivos para a humanidade a partir da nanotecnologia. Tendo o futuro como pano de fundo, a franquia Terminator coloca os humanos em conflito aberto com as máquinas, controladas por 
uma inteligência artificial, e a saga Deus Ex contrapõe bio-humanos, que aguardam a evolução natural, a trans-humanos, responsáveis pelo seu próprio trajeto evolutivo. Esta ideia de superação do "natural" domina a narrativa para suscitar eventos traumáticos, as guerras de 2027 e 2052, em Deus Ex, e 2018-2029, em Terminator, difundindo a ideia dos humanos como agentes obsoletos, e culmina numa luta pela preservação das espécies. A ideia de extermínio e genocídio, regularmente presente nos estudos da pós-memória, manifesta-se no conflito entre humanos e máquinas, em Terminator, e entre as diversas fações sociopolíticas, em Deus Ex, em particular os bio-humanos e os trans-humanos.

A ideia de "humanos obsoletos" - como se estes fossem o alvo de um processo de destruição criativa - determina uma representação do percurso de evolução que a espécie tomou, fundamentado pelo efeito Diderot que compôs a noção de unidade na aparente pluralidade trans-humana. Esta ideia de unidade consiste numa diferenciação de status dos grupos sociais e resulta da fusão da incorporação da produção tecnológica com a indumentária utilizada. No momento que os bio-humanos transitam para o estado de trans-humanos, geram novos processos culturais. Cada inovação tecnológica introduzida comporta uma mudança nas dinâmicas comportamentais.

Ambas as indústrias culturais medeiam e suavizam a temática do trauma (extermínios, genocídios e guerras) através da ação dos ecrãs e da análise destas temáticas recorrendo a cenários fictícios. A nanotecnologia como composição do processo de destruição criativa atua como catalisador de um conjunto de evoluções a partir dos humanos. Conectada com a robótica em Terminator, configura-se como o traço distintivo das máquinas, atribuindo-lhes maior resistência, velocidade e capacidade adaptativa, embutidas num sistema de transmissão e comunicação. Em Deus Ex, a nanotecnologia é integrada nos trans-humanos, melhorando as suas capacidades de regeneração, de saúde e configura a jogabilidade dos três videojogos. O cruzamento da jogabilidade com a narrativa desempenha um papel diferenciado em Deus Ex, visto possibilitar ao jogador decidir a qual das diferentes fações pretende demonstrar apoio. Esta ação de imersão proporciona ao jogador a escolha do seu trajeto individual e uma forma específica de encarar o jogo. A jogabilidade, fenómeno não exequível no cinema, confere um aspeto diferencial e constitui-se como uma das mais-valias dos videojogos. Aliar a nanotecnologia à narrativa em Terminator e à jogabilidade em Deus Ex permite a ambas as franquias explorar e desenhar um futuro e recontá-lo no presente.

Entre as inúmeras possibilidades técnicas aliadas às novas e velhas formas de desigualdade social, a ação premonitória não assenta em adivinhar se tal irá ocorrer. Visa ilustrar o que é possível ou plausível e antecipar ocorrências, atuando como pós-memória prospetiva. Crer que a nanotecnologia é apenas tecnologia, é como julgar que um vaso Ming é apenas cerâmica. 


\section{Referências bibliográficas}

ADICHIE, Chimamanda (2007), The Danger of a Single Story [Online], Disponível em: https:// youtu.be/D9Ihs241zeg [consultado em: 3 fevereiro 2016].

AGHION, Philippe, HOWITT, Peter (2006), “Joseph Schumpeter Lecture Appropriate Growth Policy: A unifying framework", Journal of the European Economic Association, 4 (2/3), pp. 269-314.

ALLEN, Richard. (1993), "Representation, Illusion, and the Cinema”, Cinema Journal, 32 (2), pp. $21-48$.

ARENDT, Hannah (2006), Eichmann in Jerusalem: A Report on the Banality of Evil, London, Penguin Classics.

BALABKINS, Nicholas W. (2003), "Adaptation Without Attribution? The Genesis of Schumpeter's Innovator” in J. Backhaus (ed.), Joseph Alois Schumpeter: Entrepreneurship, Style and Vision, New York, Springer, pp. 203-220.

BALL, Philip (2010), “Making Life: A Comment on 'Playing God in Frankenstein's Footsteps: Synthetic Biology and the Meaning of Life' by Henk van den Belt (2009)", Nanoethics, 4, pp. 129-132.

BAUMAN, Zygmunt (2007), Consuming Life, Cambridge: Polity Press.

BAUMAN, Zygmunt (2009), Does Ethics have a Chance in a World of Consumers?, Cambridge, Harvard University Press.

BAUMAN, Zygmunt (2010), Liquid Times: Living in an Age of Uncertainty, Cambridge, Polity Press.

BERBERICH, Christine (2011), “We Shall Be Punished': Positionality and Postmemory in Rachel Seiffert's The Dark Room and Uwe Timm's In My Brother's Shadow”, Holocaust Studies, 17 (2-3), pp. 261-282.

BERGHAHN, Daniela (2006), "Post-1990 screen memories: How East and West German cinema remembers the third Reich and the Holocaust", German Life and Letters, 59 (2), pp. 294-308.

CENTOLA, Damon (2015), “The Social Origins of Networks and Diffusion”, American Journal of Sociology, 120 (5), pp. 1295-1338.

CHEN, Lingchei Letty (2015), "When does 'diaspora' end and 'Sinophone' begin?”, Postcolonial Studies, 18 (1), pp. 52-66.

CHOE, Youngmin (2013), "Postmemory DMZ in South Korean Cinema, 1999-2003", Journal of Korean Studies, 18 (2), pp. 315-336.

COHEN, Anna-Lisa, GOLLWITZER, Peter M. (2008), "The Cost of Remembering to Remember: Cognitive load and the implementation intentions influence ongoing task performance", in M. Kliegel (Ed.), Prospective Memory: Cognitive, neuroscience, developmental, and applied perspectives, New York, Erlbaum, pp. 367-390.

CRICHTON, Michael (2006), Prey: A novel, London: Harper Collins Publishers.

CRUZ, Maria Teresa (2002), "Técnica e Afecção", in J. B. de Miranda e M. T. Cruz (orgs.), Ligações_Links_Liaisons, Lisboa, Tropismos, pp. 31-46.

CRUZ, Rui Vieira (2013), "Nanotecnologia e Videojogos: Contestar a narrativa do Norte a partir das Epistemologias do Sul”, Configurações, 12, pp. 199-218.

DAWESAR, Abha (2013), "Life in the 'Digital Now"” [Online], TEDTalk, disponível: https:// www.ted.com/talks/abha_dawesar_life_in_the_digital_now?language=en [consultado em: 3 fevereiro 2016].

DEBORD, Guy (1995), The Society of the Spectacle, New York, Zone Books.

DEL TORO, Guillermo (2006), El Laberinto del Fauno, Espanha, Esperanto Films. 
DIDEROT, Denis (2005), Regrets Sur ma Vieille Robe de Chambre, França, Ebooks Lib.

DONDERO, Maria Giulia, BOUKO, Catherine (2015), "My Friend is a WWI Soldier: A Semiopragmatic Approach to Docufictions on Social Networks", Punctum. International Journal of Semiotics, 1 (1), pp. 94-107.

DREXLER, K. Eric (1990), Engines of Creation: The coming era of nanotechnology, New York, Anchor Books.

DUPUY, Jean-Pierre (2010), “The Narratology of Lay Ethics”, Nanoethics, 4, pp. 153-170.

ELLIS, Warren, HITCH, Bryan (1999), The Authority, LaJolla, Wildstorm/DC Comics.

FOUCAULT, Michel (2001), Power: The essential works of Michel Foucault, vol. 3, New York, New Press.

GARCIA, José Luís (2003), "Sobre as Origens da Crítica da Tecnologia na Teoria Social”, in H. Martins e J. L. Garcia (orgs.), Dilemas da Civilização Tecnológica, Lisboa, Imprensa de Ciências Sociais, pp. 91-138.

GARCIA, José Luís (2006), “Rumo à Criação de Desenhada de Seres Humanos? Notas sobre Justiça Distributiva e Intervenção Genética”, Configurações, 2, pp. 89-101.

GARCIA, José Luís (2011), “Internet, New Forms of Power and Democracy”, Revista Internacional de Sociología, 69 (3), pp. 747-781.

GENTER, Robert (2007), “'With Great Power Comes Great Responsibility': Cold War culture and the birth of Marvel Comics", Journal of Popular Culture, 40 (6), pp. 953-978.

GERBNER, George (2012), “The stories we tell ourselves”, Journal of International Communication, 18 (2), pp. 237-244.

GIL, José (2002), “Ligação de Inconscientes”, In J. B. de Miranda e M. T. Cruz (orgs.), Ligações_ Links_Liaisons, Lisboa: Tropismos, pp. 21-30.

GÓMEZ-CASTELLANO, Irene (2013), "Lullabies and Postmemory: Hearing the ghosts of Spanish history in Guillermo del Toro's Pan's Labyrinth (El laberinto del fauno, 2006)”, Journal of Spanish Cultural Studies, 14 (1), pp. 1-18.

GORDIJN, Bert (2005), "Nanoethics: From utopian dreams and apocalyptic nightmares towards a more", Science and Engineering Ethics, 11 (4), pp. 521-533.

GRINBAUM, Alexei (2010), “The nanotechnological golem”, Nanoethics, 4 (3), pp. 191-198.

GURGA, J. J. (2012), Echoes of the Past: Ukrainian poetic cinema and the experimental ethnographic mode, Phd thesis, London, University College London.

GURGA, J. J. (2014), “Remembering (in) Ukrainian Cinema of the 1960s: Rolan Serhiienko's White Clouds (1968)", Studies in Russian and Sovietic Cinema, 5 (3), pp. 353-370.

HARAWAY, Donna (1985), "Manifesto for Cyborgs: Science, technology, and socialist-feminism in the 1980s", Socialist Review, 80, pp. 65-108.

HARTOG, François (2003), Régimes d'Historicité: Présentisme et expérience du temps, Paris, Le Seuil.

HASSAN, Robert (2010), "Social Acceleration and the Network Effect: A defence of social 'science fiction' and network determinism”, British Journal of Sociology, 61 (2), pp. 356-374.

HAYLES, N. Katherine (2006), "Unfinished Work: From cyborg to cognisphere”, Theory, Culture \& Society, 23 (7-8), pp. 159-166.

HERRMANN, Andreas, SPROTT, David, SCHLAGER, Tobias (2015), "What Drives Leapfrogging? Empirical assessment of consumer determinants of leapfrogging”, Total Quality Management \& Business Excellence, 28 (3-4), pp. 266-281.

HIRSCH, Marianne (1993), “Family Pictures: Maus, Mourning, and Post-Memory”, Discourse, 15 (2), pp. 3-29.

HIRSCH, Marianne (1997), Family Frames: Photography, narrative, and postmemory, Cambridge, Harvard University Press.

HIRSCH, Marianne (2008), "The Generation of Postmemory”, Poetics Today, 29 (1), pp. $103-128$. 
HIRSCH, Marianne, SPITZER, Leo (2006), “What'S Wrong With This Picture?”, Journal of Modern Jewish Studies, 5 (2), pp. 229-252.

HIRSCH, Marianne, \& SPITZER, Leo (2009), "Incongruous images: Before, during, and after the Holocaust", History and Theory, 48, pp. 9-25.

HOLT, Douglas B. (2002), "Why Do Brands Cause Trouble? A Dialectical Theory of Consumer Culture and Branding”, Journal of Consumer Research, 29 (1), pp. 70-90.

HUYSSEN, Andreas (2003), Present Pasts: Urban palimpsests and the politics of memory, Stanford, Stanford University Press.

JONES, Richard Anthony Lewis (2007), Soft Machines: Nanotechnology and life, New York, Oxford University Press.

KAUFFMAN, Robert J., TECHATASSANASOONTORN, Angsana A. (2012), “Guest Editors' Introduction: Methods innovations for the empirical study of technology adoption and diffusion”, Information Technology and Management, 13 (3), pp. 113-114.

KLIEGEL, Matthias, MARTIN, Mike (2003), "Prospective Memory Research: Why is it relevant?”, International Journal of Psychology, 38 (4), pp. 193-194.

KLINE, Ronald (2009), "Where are the Cyborgs in Cybernetics?", Social Studies of Science, 39 (3), pp. 331-362.

KRIPS, Henry (2010), “The Politics of the Gaze Foucault, Lacan and Žižek”, Culture Unbound: Journal of Current Cultural Research, 2, pp. 91-102.

LAAN, J. M. Van Der (2010), "Frankenstein as Science Fiction and Fact", Bulletin of Science , Technology \& Society, 30 (4), pp. 298-304.

LAKATOS, Imre (1967), "Problems in the Philosophy of Mathematics", in Proceeding of the International Colloquium in the Philosophy of Science, Amsterdam, North-Holland Publishing Company, pp. 82-96.

LAKATOS, Imre (1989), The Methodology of Scientific Research Programmes, New York: Cambridge University Press.

LORENZ, Chris (2010), "Unstuck in Time, or: the sudden presence of the past", in K. Tilmans, F. Van Vree e J. Winter (eds.), Performing the Past Memory, History, and Identity in Modern Europe, Amsterdam, Amsterdam University Press, pp. 67-105.

MARCHAND, André, HENNIG-THURAU, Thorsten (2013), "Value Creation in the Video Game Industry: Industry economics, consumer benefits, and research opportunities", Journal of Interactive Marketing, 27 (3), pp. 141-157.

MARCUSE, Herbert (1977), The Aesthetic Dimension: Toward a Critique of Marxist Aesthetics, Boston, Beacon Press.

MARCUSE, Herbert (2002), One-Dimensional Man, London, Routledge.

MARTINS, Hermínio (2003), “Aceleração, Progresso e Experimentum Humanum”, in H. Martins e J. L. Garcia (orgs.), Dilemas da Civilização Tecnológica, Lisboa, Imprensa de Ciências Sociais, pp. 19-78.

MARTINS, Hermínio (2006), "Paths to the Post-Human: A very short guide for the perplexed", Configurações, 2, pp. 29-56.

MATSUMURA, Janice (2008), The “All-Knowing” Japanese State? New scholarship on medicine, science, technology, and industrial relations, Minerva, 46 (1), pp. 77-86.

MCFARLAND, Craig, GLISKY, Elizabeth (2012), “Implementation Intentions and Imagery: Individual and combined effects on prospective memory among young adults”, Memory \& Cognition, 40 (1), pp. 62-69.

MILES, Robert J. (2011), "Reclaiming Revelation: Pan's Labyrinth and The Spirit of the Beehive”, Quarterly Review of Film and Video, 28 (3), pp. 195-203. 
MILLER, John C., SERRATO, Ruben M., REPRESAS-CARDENAS, José Miguel, KUNDAHL, Griffith (2005), The Handbook of Nanotechnology: Business, policy, and intellectual property law, New Jersey: Wiley Publishing.

MIRANDA, José Augusto Bragança de (2002), "Para Uma Crítica das Ligações Técnicas”, in J. A. B. de Miranda e M. T. Cruz (orgs.), Ligações_Links_Liaisons, Lisboa, Tropismos, pp. 259-278.

MOTOHASHI, Kazuyuki, LEE, Deog-Ro, SAWNG, Yeong-Wha, KIM, Seung-Ho (2012), "Innovative Converged Service and its Adoption, Use and Diffusion: A holistic approach to diffusion of innovations, combining adoption-diffusion and use-diffusion paradigms", Journal of Business Economics and Management, 13 (2), pp. 308-333.

MURI, Allison (2003), "Of Shit and the Soul: Tropes of cybernetic disembodiment in contemporary culture”, Body \& Society, 9 (3), pp. 73-92.

NAGAR, Komal (2015), “Consumers' Evaluation of Ad-Brand Congruity in Comparative Advertising”, Journal of International Consumer Marketing, 27 (3), pp. 253-276.

OLICK, Jeffrey K. (1999), “Collective Memory: The two cultures”, Sociological Theory, 17 (3), pp. 333-348.

ORLANDO, Valérie K. (2016), "Women, Madness, Myth and Flm: Exploring Moroccan psychological trauma and postmemory in Pegase (Mohamed Mouftakir, 2010)", The Journal of North African Studies, 21 (1), pp. 90-107.

OSARENKHOE, Aihie (2010), "A Study of Inter-firm Dynamics between Competition and Cooperation: A coopetition strategy", Journal of Database Marketing \& Customer Strategy Management, 17 (3-4), pp. 201-221.

PENG, Jing, AGARWAL, Ashish, HOSANAGAR, Kartik, IYENGAR, Raghuram (2014), "Towards Effective Information Diffusion on Social Media Platforms: An analysis of dyadic network relationship", Thirty Fifth International Conference on Information Systems, Auckland, pp. 1-45.

PEREIRA, Fernando José (2002), "Ligações Livres: Da construção fantasiosa à realidade doméstica”, in J. B. de Miranda e M. T. Cruz (orgs.), Ligações_Links_Liaisons, Lisboa,Tropismos, pp. 119-132.

PEREZ, Carlota (2007), "Great Surges of Development and Alternative Forms of Globalization”, Working Papers in Technology Governance and Economic Dynamics, 15, pp. 1-32.

PLATT, Bradley, KAMBOJ, Sunjeev K., ITALIANO, Tommaso, RENDELL, Peter G., CURRAN, H. Valerie (2016), "Prospective Memory Impairments in Heavy Social Drinkers are Partially Overcome by Future Event Simulation”, Psychopharmacology, 233, pp. 499-506.

RIBEIRO, António Sousa (2010), "Memória, Identidade e Representação: Os limites da teoria e a construção de testemunho”, Revista Crítica de Ciências Sociais, 88, pp. 8-21.

RICOEUR, P. (1984), Time and Narrative. The American Historical Review, vol. I, Chicago, University of Chicago Press.

RICOEUR, Paul (1986), Fallible Man, New York, Fordham University Press.

ROCO, Mihail C. (2007), “Convergence and Integration”, in L. E. Foster (ed.), Nanotechnology: Science, Innovation and Opportunity, New Jersey, Prentice Hall, pp. 225-232.

ROSA, Hartmut (2003), "Social Acceleration: Ethical and political consequences of a desynchronized high-speed society", Constellations, 10 (1), pp. 3-33.

ROTHBERG, Michael (2009), "Introduction: Theorizing Multidirectional Memory in a Transnational Age", in Multidirectional Memory: Remembering the Holocaust in the Age of Decolonization, Stanford: Stanford University Press, pp 1-29.

RUTTAN, Vernon W. (2010), "Usher and Schumpeter on Invention, Innovation, and Technological Change", The Quarterly Journal of Economics, 73 (4), pp. 596-606. 
SCHMIDT, Jan Cornelius (2008), "Normativity and Innovation: An approach to concepts of innovation from the perspective of philosophy of technology", Georgia Tech - Ivan Allen College Working Papers Series, 31, pp. 1-9.

SCHUMPETER, Joseph A. (1947), "The Creative Response in Economic History", The Journal of Economic History, 7 (2), pp. 149-159.

SCHUMPETER, Joseph A. (2002), "New Translations: Theorie der wirtschaftlichen Entwicklung”, American Journal of Economics and Sociology, 61 (2), pp. 405-437.

SCHUMPETER, Joseph A. (2003), Capitalism, Socialism and Democracy, London, Routledge.

SHIELDS, Rob (2006), "Flanerie for Cyborgs”, Theory, Culture \& Society, 23 (7-8), pp. 209-220.

SHIONOYA, Yuichi (2004), "Schumpeter's Preface to the Fourth German Edition of The Theory of Economic Development”, Journal of Evolutionary Economics, 14, pp. 131-142.

SHIONOYA, Yuichi (2007), Schumpeter and the Ideia of Social Science, Cambridge, Cambridge University Press.

SILVA, Sandra Tavares (2009), "On Evolutionary Technological Change and Economic Growth: Lakatos as a starting point for appraisal”, Journal of Evolutionary Economics, 19, pp. 111-135.

SMALLEY, Richard (2007), “Nanotechnology and Our Energy Challenge”, in L. E. Foster (ed.), Nanotechnology: Science, innovation and opportunity, New Jersey, Prentice Hall, pp. 13-18.

STEPHENSON, Neal (1995), The Diamond Age, New York, Bantam Spectra.

SWEDBERG, Richard (2002), "The Economic Sociology of Capitalism: Weber and Schumpeter”, Journal of Classical Sociology, 2, pp. 227-255.

TARKOVSKY, Andrei (1979), Stalker, URSS, Mosfilm.

TENENBOIM-WEINBLATT, Keren (2013), "Bridging Collective Memories and Public Agendas: Toward a theory of mediated prospective memory", Communication Theory, 23 (2), pp. 91-111.

TOUMEY, Chris (2011), "Seven Religious Reactions to Nanotechnology”, NanoEthics, 5 (3), pp. 251-267.

VALENZUELA, Sebastián, BRANDÃO, Angela S. (2015), "Historical Dramas, Current Political Choices: Analyzing partisan selective exposure with a docudrama", Mass Communication and Society, 18 (4), pp. 449-470.

VELTRI, Giuseppe A., ATANASOVA, Dimitrinka (2015), "Climate Change on Twitter: Content, media ecology and information sharing behaviour", Public Understanding of Science, (November online first), pp. 1-17.

VIOLANTE, Giovanni L. (2002), "Technological Acceleration, Skill Transferability, and the Rise in Residual Inequality", The Quarterly Journal of Economics, 117 (1), pp. 297-338.

WARREN, Adam, MAYS, Rick (2005), Livewires, New York, Marvel Comics.

WASSERMAN, Brooke (2015), Valuation of Intangible Assets: Should brand equity be accounted for on the balance sheet?, Honors Scholar Theses, Storrs: University of Connecticut.

ZIMMER, Catherine (2011), "Surveillance Cinema: Narrative between technology and politics", Surveillance \& Society, 8 (4), pp. 427-440. 